\title{
LE CONDITIONNEL EN DZUUNGOO DE SAMOGOHIRI
}

\author{
Paul Solomiac \\ SIL Burkina Faso
}

\begin{abstract}
L'examen des phrases conditionnelles du dzùùngoo et des différents encodages de l'expression de la condition et de l'irréel révèle dans cet article une grande variété de structures et de sens. L'auteur fait le tour des différents types de constructions associés au morphème du conditionnel, ainsi qu'aux différents types d'encodage à l'expression de la condition.
\end{abstract}

Keywords : conditional, Dzùùngoo, Niger-Congo

Le dzùùngoo (de code ISO 639-3, dnn) est une langue de la grande famille Niger-Congo, du groupe mandé, et plus particulièrement de la branche mandé-ouest, nord-ouest selon la classification la plus récente de Raimund Kastenholz (Kastenholz 2003). Comme toutes les langues mandé, les noms ne sont pas regroupés en genres ou classes et la phrase verbale simple du dzùùngoo a la structure générale SOV ; entre les paradigmes du sujet et de l'objet, un paradigme est réservé à des particules prédicatives (pp), marques du mode (dont le conditionnel), du temps ou de l'aspect, de la négation ou des auxiliaires. Les quatre schèmes de prédication de phrases simples non verbales (phrase à prédicat nominal, adjectival, locatif ou copulaire) présentent, eux aussi un paradigme de particules prédicatives (pp) qui suit immédiatement le paradigme du sujet. Le paradigme pp est aussi celui des particules de négations ; toutefois l'expression de la négation nécessite encore l'intervention de morphèmes en position finale de phrase. L'expression de la condition renvoyant à la problématique des phrases complexes, on notera enfin que le dzùùngoo utilise peu les conjonctions de subordination (Solomiac 2014).

\section{La phrase conditionnelle}

On reconnaît en dzùùngoo une structure complexe de phrases subordonnées qu'on qualifiera ici de conditionnelles. Même si, sémantiquement, ces phrases ne font pas toutes état explicitement d'une condition, elles partagent trois caractéristiques structurelles qui permettent de les distinguer des autres structures complexes de subordination :

- $\quad$ Elles présentent un ordre plutôt fixe P protase - Q apodose ;

- La protase $\mathrm{P}$, subordonnée à l'apodose, ne présente pas de conjonction de subordination ;

- La protase $\mathrm{P}$ est caractérisée par le morphème libre du mode conditionnel (glosé COND) et l'apodose Q est caractérisée par le mode indicatif ou subjonctif.

Dans le système verbal du dzùùngoo, le morphème du conditionnel est considéré comme une particule modale plutôt que comme une particule de temps grammatical. Il est en effet associé à l'expression de l'irréel ou de l'hypothétique plutôt qu'à des catégories temporelles ou aspectuelles. 
Il partage en plus avec le morphème modal du subjonctif (glosé SUB) plusieurs caractéristiques communes qui le qualifient parmi les catégories verbales comme un mode :

- Morphologiquement, les deux présentent deux formes libres comparables, une pour l'affirmatif et une pour le négatif :

\begin{tabular}{l|l|l} 
& SUB & COND \\
\hline affirmatif & yè & $\mathrm{ye}^{1}$ \\
\hline négatif & mà & ma
\end{tabular}

- Tous deux appartiennent à la phrase complexe et plus particulièrement à la subordonnée.

Un examen rapide des emplois du morphème du conditionnel permet de remarquer que les constructions complexes, que nous avons appelées phrases conditionnelles, dans lesquelles il apparaît, présentent une grande variété d'usages différents. Le dzùùngoo exprime en effet l'irréel, l'hypothétique, l'éventuel, le potentiel au travers de constructions syntaxiques complexes de structure $\mathrm{P}-\mathrm{Q}$ dans lesquelles le morphème COND (ou COND-NEG) apparaît dans la protase $\mathrm{P}$, dans le paradigme des particules dites de prédication (pp) et des auxiliaires situé entre celui du sujet (S) et celui de l'objet (O) ou du verbe (V) pour les constructions intransitives : S pp (O) V.

\begin{tabular}{|c|c|c|c|c|c|c|c|}
\hline & $\mathrm{P}$ & & /1 & & $\mathrm{Q}$ & & \\
\hline S & $\mathrm{pp}$ & $\mathrm{V}$ & /1 & $\mathrm{S}$ & $\mathrm{pp}$ & $\mathrm{V}$ & \\
\hline tàan & rèè & ye & dzyéè & mòó & dziá & wó & è $\mathrm{ra}$ \\
\hline femme & PL & COND & réunir_IAC // & personne & pouvoir_IAC & aider & 3PL à \\
\hline
\end{tabular}

Dans la phrase conditionnelle ci-dessus, le formant $\mathrm{P}$, la protase, pose un procès à réaliser, celui de se réunir comme condition explicite à la réalisation du formant $\mathrm{Q}$, l'apodose, auquel il est subordonné. Mais toutes les phrases conditionnelles que nous avons relevées dans les textes dzùùngoo n'expriment pas forcément ce type de condition aussi explicite.

1 Le dzùùngoo est une langue tonale à trois tons, haut $(\mathrm{H})$, transcrit avec un accent aigu sur la voyelle, moyen (M), pas transcrit, et bas (B), transcrit avec un accent grave sur la voyelle. Les deux morphèmes du conditionnel sont à ton $\mathrm{M}$.

2 La plupart des exemples illustratifs de cet article ont été tirés d'une sélection de 10 textes naturels parmi les plus longs et mieux construits de notre base de données textuelle ; il s'agit de textes essentiellement narratifs, mais aussi des conversations et des chants traditionnels collectés entre 1994 et 2005 à Samogohiri et Saraba auprès d'une variété de conteurs et de chanteurs; entre parenthèses, le titre et le numéro de ligne par lesquels ils ont été indexés. Les exemples qui ne sont pas référencés ont été sollicités avec un informateur. 


\begin{tabular}{lllllll} 
(2) gè & \multicolumn{1}{c}{ gé } & ye bá wò raa & tsú \\
chose tout & chose & COND devenir LOG & POS & semence \\
twèy mà & bè & donkos dón & $\mathrm{d} \varepsilon$ & \\
DÉM SUB-NÉG & aller & marigot dans plus &
\end{tabular}

'Toutes celles qui sont de sa descendance, qu'elles n'aillent plus au marigot.' (Mariage 29)

La phrase de ce deuxième exemple est impossible à traduire en français par une phrase en 'si' ; la protase, c'est-à-dire la première ligne, est une proposition non verbale à prédicat nominal ; le syntagme nominal distributif avec le rôle syntaxique de sujet l'apparente plutôt à une relative qui donnerait un cadre restrictif référentiel au rôle syntaxique sujet de l'apodose de la deuxième ligne.

Les classifications existantes des constructions conditionnelles s'entendent toutes pour distinguer entre deux valeurs fondamentales, le réel et l'irréel ; Jack Feuillet (Feuillet 2006) résume cette distinction en posant :

... une césure fondamentale entre ... le realis et l'irrealis correspondant en gros à une opposition virtualité d'actualisation / virtualité de distanciation. [p. 520]

Les 196 constructions conditionnelles du dzùùngoo que nous avons rencontrées dans notre corpus font en effet état d'une virtualité pour laquelle on peut distinguer des formes plus associées à l'actualisation et d'autres plutôt à la distanciation. Feuillet propose une grille générale modulable, adaptable ou à affiner selon les langues avec trois zones de pénétration dans l'hypothétique :

- Une zone indicative réservée à la réalité :

○ Équatif : si $\mathrm{x}=2$, alors $2 \mathrm{x}=4$;

○ Temporel, causal (si équivalent à comme, puisque, étant donné que...) : si c'est comme ça, je m'en vais ;

○ Protase assumée comme réelle : si tu vas en ville, rapporte-moi le journal ;

- Une zone transitoire, intermédiaire entre la réalité et l'hypothétique, réservée au doute :

○ Éventuel : s'il fait beau, je sors ;

○ Potentiel : si je peux, je viendrai ;

- Une zone hypothétique réservée à l'irréel et pour laquelle on peut distinguer entre :

○ L'irréalisable : si j'avais des ailes, je volerais ;

○ L'irréalisé : si j'avais su, je ne serais pas venu.

En suivant et en adaptant le plan proposé par Feuillet, nous présenterons premièrement les différents types de constructions ou phrases conditionnelles recensées dans notre corpus, en allant de ce qui relève plus de la réalité vers l'expression de l'irréel. Dans un second temps, nous passerons en revue les constructions conditionnelles qui ne respectent pas les 3 caractéristiques communes à la grande majorité d'entre elles, c'est-à-dire, celles qui ne respectent pas l'ordre protase - apodose, celles qui utilisent une conjonction de subordination et celles qui n'utilisent pas le morphème du conditionnel. 


\section{Les constructions conditionnelles typiques}

2.1. Les réelles causales. Un premier type de condition fréquent dans les narrations pourrait être paraphrasé par une causale ; on pourrait remplacer le si de la traduction en français par un comme, un puisque ou un étant donné que.

(3)
shi fon'ná ye bá
route repas_DÉF COND devenirCOP 2SG poche // 2SG aller_IAC
'... si tu as le titre de transport en poche, tu y vas.' (Sensibilisation 29 )

La première proposition de cette phrase, la subordonnée conditionnelle, exprime autant une condition qu'une cause à la principale qui la suit.

2.2. Les assumées réelles ou temporelles. Un deuxième type, encore plus fréquent dans les narrations pourrait être comparé à une temporelle.

(4) $\begin{array}{llllll}\mathrm{e}^{3} & \text { byan } & \mathrm{ji} & / / & \mathrm{e} & \text { tò flá } \\ \text { 3SG_COND } & \text { crotte } & \text { mettre } & / / & 2 \mathrm{SG} \text { DÉM balayer }\end{array}$
'Si $(\approx$ quand)
il dépose ses crottes, tu les balaies.' (Colonisation 170)

Certaines de ces subordonnées conditionnelles (4 occurrences dans notre corpus) n'ont d'autre fonction que de situer le procès de la principale dans le temps.

$\begin{array}{llllllll}\text { (5) dzò̀n } & \text { ye } & \text { tsí } / / & \text { è nà } & \text { dòn mìn féne } \\ \text { sol_DÉF } & \text { COND } & \text { noircir // } & \text { 3PL venir } & \text { entrer } & \text { ici } & \text { nuit }\end{array}$

'Dès qu'il fait sombre (lit. si le sol devient sombre), ils entrent ici de nuit.' (Colonisation 97)

La subordonnée conditionnelle de l'exemple ci-dessus exprime le crépuscule, la tombée de la nuit ; l'aurore, le levé du jour a aussi une telle expression analogue :

$\begin{array}{lll}\text { (6) dzò̀n } & \text { ye } & \text { vi } \\ \text { sol_DÉF } & \text { COND } & \text { éclairer }\end{array}$

'Dès qu'il fait clair (lit. si le sol s'éclaire).'

Le dzùùngoo possède ainsi un nombre limité de subordonnées conditionnelles qui lui tiennent lieu de formules temporelles ; nous avons relevé dans les textes de notre corpus limité :

3 Le pronom sujet non emphatique est normalement amalgamé avec le morphème du conditionnel ou du subjonctif affirmatif ; les amalgames pronom_pp suivants apparaissent dans les exemples de cet article :
à + yē $\rightarrow$ è $/ \overline{\mathrm{a}}+\mathrm{ye} \rightarrow \overline{\mathrm{e}} / \quad$ à + yè $\rightarrow$ è $/ \overline{\mathrm{a}}+$ yè $\rightarrow \overline{\mathrm{e}} / \quad$ é + yè $\rightarrow$ yē
$3 \mathrm{~S}$ COND / $2 \mathrm{~S}$ COND / $3 \mathrm{~S}$ SUB $/ 2 \mathrm{~S}$ SUB $/ 2 \mathrm{P}$ SUB
mún + yē $\rightarrow$ mín
1s COND 
(7) a. jí ye sééy

aube COND faire jour

'le lendemain (lit. si l'aube point)'

b. tò síi sèè ye wó

DÉM jour retour COND arriver

'ce jour-là (lit. si ce jour arrive)'

c. tò watsiá ye dzí

DÉM moment_DÉF COND arriver

'à ce moment-là (lit. si ce moment arrive)'

À côté de ces subordonnées qui ont une forme presque figée, on trouve aussi des subordonnées conditionnelles associées (6 occurrences dans notre corpus) à un nombre limité de formules relatives elles aussi figées (avec le relatif riì ou niì glosés REL):
a. síí rìi sèè
jour REL retourner
'le jour où...'
b. sií nií sèè
jour REL retourner
variante de a.
c. watsi ríi nàn
moment REL dans
'au moment où...' moment RELjour variante de $\mathrm{c}$.
d. watsi ríi bwèy

On ajoutera encore que ces formules sont plus fréquentes dans l'expression du temps dans des phrases à l'indicatif que dans les subordonnées conditionnelles. Ces phrases conditionnelles pourraient être mises à l'indicatif sans en changer le sens. On ne peut que supposer que le narrateur a voulu y ajouter une nuance d'incertitude. Pour certaines occurrences, cette nuance n'est pas évidente.

$\begin{array}{lllllll}\text { (9) kààmòò } & \text { kpír'là } & \text { ci } & \text { yé }^{4} & \text { e } & \text { tsúù miín } \\ \text { marabout } & \text { homme } & \text { que } & 2 \mathrm{PL} & \text { RÉF } & \text { poser } & \text { ici }\end{array}$

síč ye bá ni síi rìi sèè

marché COND devenirCOPjour REL revenir

yé síi gur'lá tsi ná $\mathrm{n}$ ko

2PL marché taxe_DÉF prendre venir 1SG pour

'Le marabout dit : "Installez-vous ici ; le jour où il y a marché, prenez la taxe sur les marchandises pour moi."' (Jiri-2 166)

4 Quelques pronoms personnels du dzùùngoo ont deux formes, une forme brève non emphatique et une forme plus longue emphatique; les formes suivantes se rencontrent dans cet article :

$1 \mathrm{~s}:$ mún $=\mathrm{n}$ emphatique $; 2 \mathrm{~s}:$ wó $=\overline{\mathrm{a}}$ emphatique $; 2 \mathrm{p}:$ yé $=$ é emphatique. 
L'exemple ci-dessus relatant une invitation, c'est-à-dire un procès qui n'appartient pas au domaine du réel, mais d'un potentiel à envisager, le morphème du conditionnel vient en souligner l'incertitude ; il est juste surprenant que la condition porte sur la proposition qui ne présente justement pas d'incertitude.

2.3. Les relatives. Alors que les caractéristiques aspecto-modales de la proposition relative dzùùngoo sont plutôt indicatives, on rencontre dans les textes des exemples de propositions relatives marquées par le morphème du conditionnel ( 8 occurrences dans notre corpus).

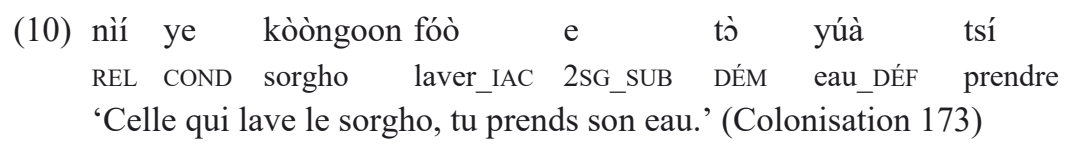

La proposition relative de la phrase ci-dessus serait grammaticalement correcte sans le morphème du conditionnel avec un même sens pour la phrase. Le morphème ye donne à la phrase une valeur d'incertitude qui peut être associée au mode conditionnel, "celle qui laverait le sorgho...". Cette phrase du texte suit d'ailleurs une phrase conditionnelle avec une apodose identique :

$\begin{array}{llllllll}\text { (11) tàan } & \text { rèè ye } & \text { bá fiidzin fóò } & \text { e } & \text { tò } & \text { yúà } & \text { tsí } \\ \text { femme } & \text { PL } & \text { COND } & \text { devenir fonio laver_IAC } & \text { 2SG_SUB } & \text { DÉM } & \text { eau_DÉF } & \text { prendre }\end{array}$

'Si les femmes lavent le fonio, tu prends cette eau-là.' (Colonisation 172)

On peut poser que ces constructions relatives atypiques, marquées par le morphème du conditionnel, le sont pour indiquer à l'interlocuteur une nuance d'incertitude dans la limitation référentielle signifiée par la relative. On le perçoit nettement dans l'exemple suivant traduit spontanément en français avec un conditionnel par l'auteur du texte :

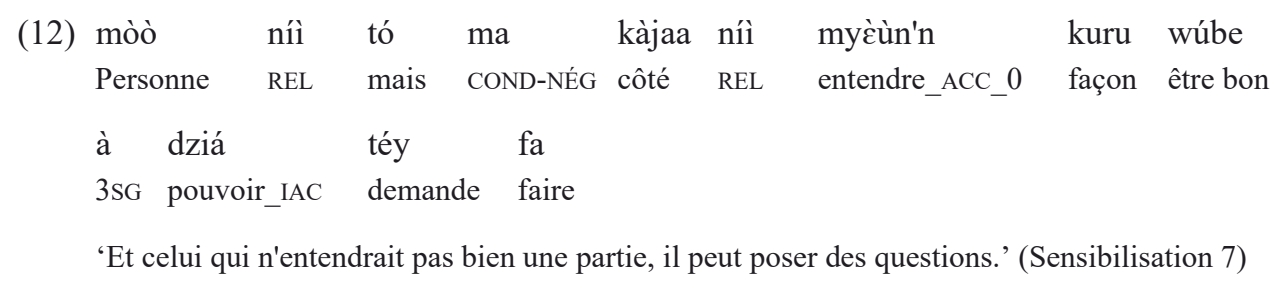

2.4. Une forme de thématisation : l'introduction d'un thème. Le lien entre conditionnel et thématisation a déjà été évoqué (Haiman 1978). Or, une construction fréquente (29 occurrences dans notre corpus) commune autant au dialogue qu'à la narration ou à l'exposé, et dans laquelle l'énonciateur signale à son interlocuteur qu'il introduit un nouveau thème, un nouveau point dans son discours, est facilement reconnaissable par la forme de la protase. Dans la plupart des cas, la protase de ces constructions consiste en une séquence principale - complétive dans laquelle le verbe de la principale est un verbe de perception (voir, entendre...), la complétive contient le nouvel argument introduit, et l'apodose valide le nouvel argument. 


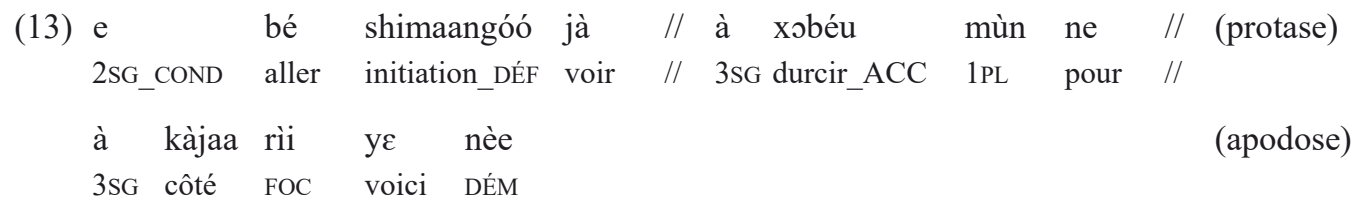

'Si tu vois que l'initiation a été difficile pour nous, c'est de cela qu'il s'agit (lit. c'est son côté que voici).' (Mariage 52)

Il arrive aussi, quoique moins fréquemment, que l'objet du verbe de perception ne soit pas une complétive mais un constituant nominal :

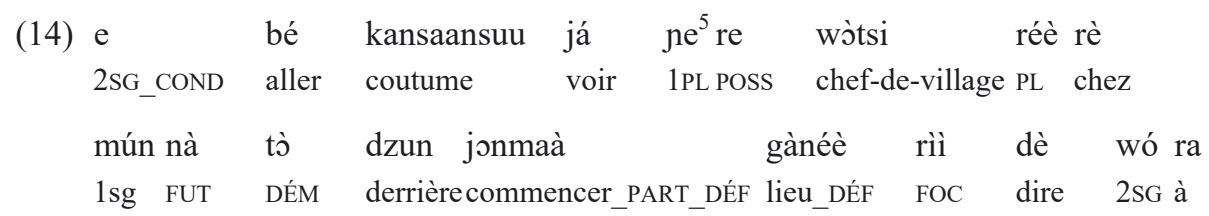

'Si tu vois (cette coutume appelée) "kassassou" chez nos chefs, c'est le commencement de cette histoire que je vais te dire maintenant.' (Jiri-2 46)

L'énonciateur peut utiliser cette même construction pour clore le point qu'il vient de traiter et qu'il avait ouvert de la même façon ; ainsi, l'exemple précédent constitue l'introduction d'un point dans le discours ; l'exemple suivant en constitue la clôture.
(15) a y $y^{\prime} a^{6}$ jà ci kansaansuu yéè bà ni ne re wòtsi réè rè 2SG COND_3SG voir que coutume SUB devenirCOP1PL POSS chef-de-village PL chez àwà ta tò gànéè rì̀ éh bien DÉM DÉM lieu_DÉF FOC
'Si tu vois que (cette coutume appelée) "kassassou" est chez nos chefs, éh bien, c'est de ce point qu'il était question.’ (Jiri-2 55)

L'apodose de telles constructions peut, en plus de valider le nouveau thème, commencer à en faire la présentation.

\footnotetext{
5 En dzùùngoo, on distingue entre deux pronoms $1^{\text {ère }}$ personne du pluriel, un inclusif mùn et un exclusif $n \bar{e}$.

6 Dans une séquence pronom sujet - conditionnel / subjonctif - pronom objet, l'amalgame se fait entre la particule modale et le pronom objet; dans l'orthographe du dzùùngoo, l'apostrophe symbolise l'élision de la voyelle /e/ qui intervient lors de l'amalgame ; on notera que le verbe bè "aller" en position auxiliaire subit le même amalgame ; les amalgames suivants se rencontrent dans cet article :

$$
\begin{aligned}
& y \bar{e}+\text { à } \rightarrow y^{\prime} \bar{a} / y \bar{e}+\bar{a} \rightarrow y^{\prime} \bar{a} / \text { bè }+ \text { à } \rightarrow \text { b'à } \\
& \text { COND 3SG / COND 2SG / aller 3SG }
\end{aligned}
$$


$\begin{array}{lllllllll}\text { (16) a } & \text { y'a } & \text { myc̀n } & \text { ci jónà } & / / & \text { è sá } & \text { ko e shí ríi ni } \\ \text { 2SG } & \text { COND_3SG } & \text { entendre } & \text { que initiation } & / / & \text { 3PL tous } & \text { et } & \text { RÉF route } & \text { FOCCOP }\end{array}$

'Si tu entends initiation, il en existe plusieurs sortes (lit. elles sont toutes chacune avec sa route).' (Mariage 5)

2.5 Une autre forme de thématisation : le tête-à-queue. Dans une narration, le conteur peut placer une sorte de pause dans une séquence de procès qu'il relate ; un des procès dans la chronologie des évènements est topicalisé sous la forme d'une reprise avant de poursuivre avec la séquence. Cette reprise est toujours caractérisée par le morphème du conditionnel.

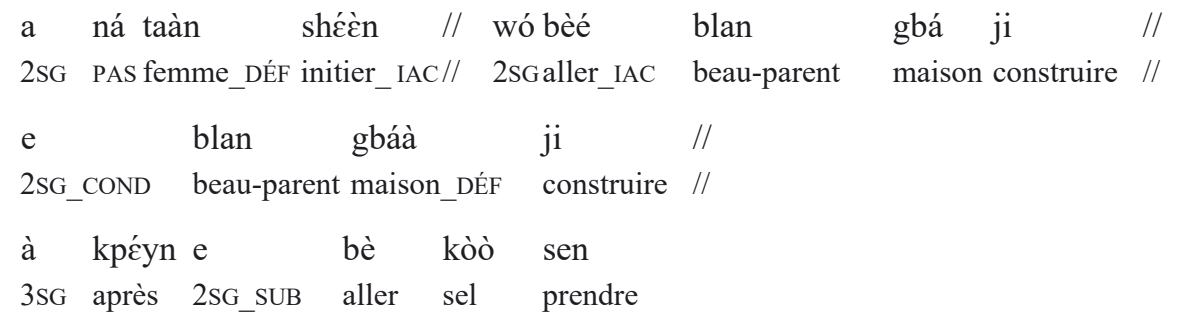

'... Pour marier une femme, toi, tu vas construire une maison pour les beaux-parents. Si tu as construit la maison des beaux-parents, après ça, tu vas prendre du sel.' (Mariage 54-55)

Dans l'exemple ci-dessus, le topique est placé sur 'la construction de la maison des beauxparents' ; le formant de la phrase de la première ligne, qui la relate, est repris dans la phrase de la ligne suivante sous forme d'une subordonnée conditionnelle. On pourrait traduire cette partie de la phrase "ayant construit la maison...". Cela dit, le procédé narratif n'est pas très productif puisque nous n'en avons relevé que quatre occurrences dans tout notre corpus.

2.6. Les "conditionnelles distributives". Le dzùùngoo a une structure spéciale de syntagme nominal coordinatif appelé SN distributif (Solomiac 2014, p 227) ; nous avons relevé dans notre corpus les exemples suivants de tels SN distributifs associés à une subordonnée conditionnelle :
(18) a. gè o gé
'Toutes les choses (lit. chose tout chose).'
b. $\min$ o $\min$
'Partout, ou n'importe où (lit. où tout où)'
c. gooma o gooma 'Toutes les paroles (lit. parole tout parole).'
d. goo o goo 'Toutes les affaires (lit. affaire tout affaire).'
e. mòò o móò 'Tout le monde (lit. personne tout personne).'
f. dzíi o dzíí 'Autant (lit. nombre tout nombre).'

Dans le contexte d'une subordonnée conditionnelle, cette structure distributive permet d'exprimer une variation à un adverbe, un nom ou un interrogatif : 
(19) Un adverbe :

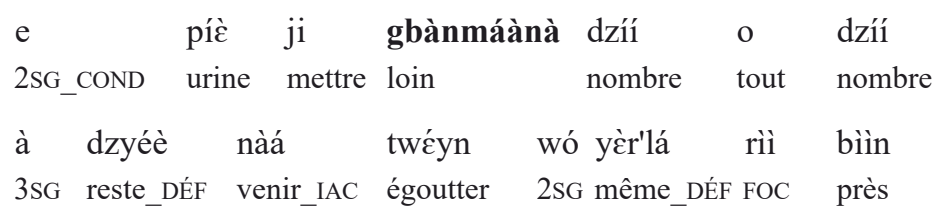

'Aussi loin que tu projettes ton urine, le reste vient goutter juste devant toi.' (Proverbe 23)

(20) Un nom :

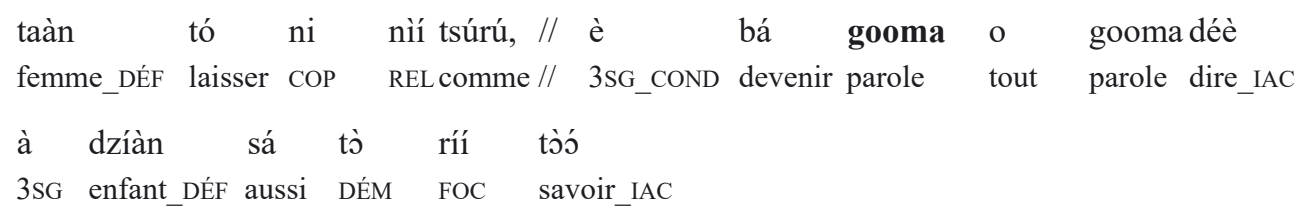

'Mais la femme est ainsi faite que quelque langue qu'elle parle, son enfant aussi connaît cela.' (Jiri-2 68)

(21) Un interrogatif:

kúrú e bá ná bèé min o min // vwèc̀yràge na ni waa hier 2SG_COND devenirPAS aller_IAC où tout où véhicule NÉG COP NÉG 'Autrefois, où que tu ailles, il n'y avait pas de véhicule.' (Sensibilisation 25)

La nature distributive du SN qui caractérise ces conditionnelles rend impossible leur traduction en français par une phrase en 'si'. Il peut être intéressant de noter que Maurice Grévisse parlait, à propos du français, de propositions concessives qui expriment un fait variable ; un terme appartenant à ces propositions est détaché en tête de la proposition et rappelé par le pronom relatif que (Grévisse, 1986, p1669, §1092). Grévisse distinguait aussi trois cas pour cet élément détaché, un adjectif (ou un adverbe), un nom ou un interrogatif :

(22) a. aussi loin que tu partes, je te retrouverai.

b. quelque promesse que tu fasses, je ne te croirai pas.

c. où que tu ailles, je te retrouverai.

À la différence du français, l'élément de la phrase mis en variation en dzùùngoo n'est pas antéposé mais reste à sa position syntaxique normale ; mais comme en français, ce type de subordonnée, qu'on la qualifie de concessive ou de conditionnelle, est associé à un mode non indicatif.

2.7. Les éventuelles - potentielles. En s'éloignant de la zone du réel, pénétrant dans la zone du douteux, on rencontre d'autres types de conditions liées aux notions d'éventualité ou de possibilités ; s'y expriment les vœux, laissés à la volonté de Dieu : 
(23) è dzibe yááblà ra // gàné rèe nà bà gàné nencnma ní 3SG_COND plaire Dieu à // lieu DÉM FUT devenirlieu bien FOC 'S'il plait à Dieu, cet endroit sera un bon endroit.' (Jiri-2 29)

Faute d'encodage spécial pour aucune de ces nuances, il n'est pas aisé de distinguer entre les nuances d'éventuel et de potentiel :

$\begin{array}{lllll}\text { (24) yà } & \text { mún } & \text { ye } & n \text { tsúù } & \text { wó ka } \\ \text { mais } & 1 \mathrm{SG} & \text { COND } & \text { RÉF poser_IAC } & 2 \mathrm{SG} \text { avec }\end{array}$

mún nà nèe bà $\mathrm{n}$ ne jaaraví faá jwćyn nì

1SG FUT DÉM devenir RÉF POS fête faire_IAC nourriture FOC

'Mais si je m'installe avec toi, je ferai de ça mon plat de fête.' (Jiri-2 48)

2.8. Les concessives. Une concessive indique une opposition, une restriction par rapport à la proposition de la principale. Une concessive n'exprime pas vraiment une condition, mais en dzùùngoo, elle est caractérisée par le morphème du conditionnel.

(25) fà kènsecn ye ji tsè dzúón nàn

folie même-si COND mettre poule œuf_DÉF dans

à na dzía e kóròngoron fáà tse waa

3SG NÉG pouvoir_IAC RÉF rouler latérite_DÉF sur NÉG

'Même si l'œuf de poule devient fou, il ne peut pas faire des roulades sur la latérite.' (Proverbes 71)

C'est le morphème kènscen glosé "même si", qui appartient, comme le morphème du conditionnel, au paradigme pp des particules prédicatives et des auxiliaires, qui distingue une concessive d'une autre conditionnelle.

On doit encore signaler à propos des concessives, un emploi fréquent emprunté au jula, la langue véhiculaire de toute la moitié ouest du Burkina Faso. Les locuteurs de la langue étant très largement bilingues en jula, beaucoup ont tendance à calquer les usages et les structures syntaxiques de cette langue à la leur ; ainsi, selon l'âge et le degré de familiarité du locuteur avec sa langue seconde, la conjonction de subordination jula hali de la concessive est fréquemment utilisé pour construire des concessives en dzùùngoo.

(26) árì wó mà bà kéréo ye

même_Si 2SG SUB-NÉG devenir accoucher_RÉT encore

mún na nà wó tó she waa

$1 \mathrm{SG} \quad$ NÉG FUT 2SG laisser aujourd'hui NÉG

'Même si tu n'as pas encore accouché, je ne vais pas te laisser aujourd'hui.' (Buffle 24)

En plus de la conjonction árì, ce type de subordonnée concessive se distingue de la concessive authentiquement dzùùngoo par l'emploi du mode subjonctif au lieu du conditionnel. 
2.9. Les irréelles ou hypothétiques. L'examen de ce que nous avons appelé les constructions conditionnelles permet de distinguer formellement entre l'expression du réel et de l'irréel et c'est au niveau de l'apodose que s'effectue cette distinction formelle ; la combinaison du morphème du passé ná associé au morphème du futur nà donne à l'apodose un sens d'irréel. On peut affirmer que la séquence des deux morphèmes du passé et du futur caractérise la zone de l'hypothétique, qu'il soit irréalisé ou irréalisable.

$\begin{array}{llllllll}\text { (27) wó ye } & \text { bá ná nàu } & \text { à dè } & \text { mún } & \text { na } \\ \text { 2SG } & \text { COND } & \text { devenir PAS venir_ACC } & 3 \mathrm{SG} \text { dire } & 1 \mathrm{SG} & \text { à } \\ \text { mún ná } & \text { nà } & \text { neyn sín } & \text { wó } & \text { ra } & & \\ \text { 1SG } & \text { PAS } & \text { FUT } & \text { argent donner } & 2 \mathrm{SG} & \text { à } & & \end{array}$

'Si tu étais venu me le dire, je t'aurais donné de l'argent.' (La tromperie 68)

L'exemple ci-dessus illustre un hypothétique irréalisé ; l'exemple suivant, tiré d'un chant d'initiation illustre un hypothétique irréalisable.

(28) mòò ye bá ná dè vú naan cinaán we personne COND devenir PAS dire voix mal échanger_IAC eh! mún ná nà bè $\mathrm{n}$ naa cinan 1S PAS FUT aller 1SG POSS échanger

'si l'on échangeait une mauvaise destinée hé, j'irais échanger la mienne.' (Chant 18 de Zantsi, 007)

Dans le même texte narratif de l'exemple (27), quelques lignes plus haut, on trouve des exemples de phrases simples, non marquées par le morphème du conditionnel, qui utilisent la même séquence morphèmes du passé et du futur avec le sens irréel de projection dans le futur d'une situation évoquée dans le passé. Le personnage d'un conte explique à son ami comment il en est arrivé à le tromper en lui vendant un récipient plein de gravier pour un récipient de miel; il se justifie en lui disant :

(29) mún fá ni dzí à dè wó jaara sí síi ron// ci à byééscyn ni waa // 1SG FUT-NÉG COPpouvoir 3SGdire2SGœil marché à // que 3SGgravier FOCNÉG // $\begin{array}{lllllllll}\text { kàtsu } & \text { mún } & \text { ne } & \text { síê } & \text { ná nà bara } & / / & \text { mún } & \text { ná nà sééy tàrà } \\ \text { parce-que } & 1 \mathrm{SG} & \text { POSS } & \text { marché_DÉF } & \text { PAS FUT gâter } & / / & 1 \mathrm{SG} & \text { PAS FUThonte gagner }\end{array}$ 'je n'aurais pas pu t'avouer en face au marché que c'est du gravier. En effet, mon marché allait se gâter. J'allais avoir honte.' (La tromperie 61 à 63)

Les deux phrases de la deuxième ligne de cet exemple pourraient être reformulées en français au mode conditionnel : "En effet, mon marché aurait été gâté. J'aurais eu honte."

La combinaison des morphèmes du passé et du futur dans l'apodose, marque complexe de l'irréel en dzùùngoo, présente dans les textes quelques variations ; si l'apodose se trouve être à la 
négative, c'est la combinaison de fá, la variante négative du morphème du futur avec ni, la copule, qui est utilisée :
(30) mín
bá ná à tòu
// mún fá
ni ná
waa
1SG_COND devenir PAS 3SG Savoir_ACC // 1SG FUT-NÉG COPvenir NÉG
'Si je l'avais su, je ne serais pas venu.'

On aura remarqué dans l'exemple (29) ci-dessus que c'est la même combinaison de morphèmes qu'on retrouve à la première ligne traduite en français au mode conditionnel.

Dans un des textes de notre base de données, le conteur choisit une forme verbale fléchie à l'accompli et à l'inachevé pour dire la même chose qu'en (30) qui est une phrase sollicitée à un informateur.

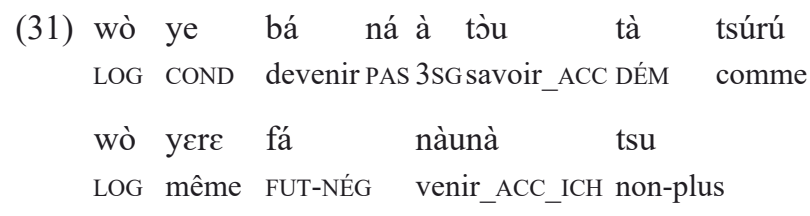

'S'il l'avait su, lui-même ne serait pas venu non plus.' (Musa-3 39)

Il existe une autre construction conditionnelle qui exprime l'irréel et dont l'apodose ne convoque pas la séquence des deux morphèmes du passé et du futur ; il s'agit d'une construction avec la postposition bóshòùn "sauf, sans", rencontrée dans un proverbe.

$\begin{array}{lllll}\text { (32) à } & \text { ma } & \text { bá } & \text { danga } & \text { bóshòùn } \\ \text { 3SG } & \text { COND-NÉG } & \text { devenir } & \text { malédiction } & \text { sans }\end{array}$

ymánín dziá sćèn tsì̀ma sen

hérisson pouvoir_IAC porc-épic_DÉF place prendre

'Si ce n'était pas à cause de la malédiction, le hérisson aurait pu prendre la place du porcépic.' (Proverbes 86)

Cette phrase conditionnelle présente un verbe à l'inaccompli dans son apodose, alors qu'elle exprime une affirmation irréalisable bien traduite en français au mode conditionnel.

$\mathrm{Au}$ niveau de la forme de la protase, la quasi-totalité des cas de conditions hypothétiques présentent une même construction en position pp des particules prédicatives et des auxiliaires, ye bá ná 'COND devenir PAS' ainsi qu'on peut le constater à la première ligne des exemples (27) à (31) ci-dessus. Un texte poétique de notre base textuelle présente une protase sans morphème du conditionnel mais juste l'auxiliaire bà "devenir". 
(33) dzunweynbé bà fîrá gè we jeunesse deveniracheter_IAC chose eh! mún ná nà bè fì à re 1SG PAS FUT aller acheter 3 SG chez

'Si la jeunesse était une chose à acheter, j'irais en payer chez lui.' (Tata-01 38)

On peut penser qu'il s'agit ici d'une ellipse propre au langage poétique ; ces vers appartenant à une chanson, il est possible que le ye du conditionnel ait constitué une syllabe de trop dans la phrase mélodique ; l'auxiliaire bà "devenir" étant familier de l'expression de l'incertitude dans d'autres constructions, et la séquence ná nà "passé, futur" signalant l'irréel dans l'apodose, la phrase pouvait se passer du morphème du conditionnel.

\section{Les phrases conditionnelles hors norme}

À côté des variantes de constructions conditionnelles que nous venons de présenter, on rencontre encore quelques phrases qui ne satisfont pas à l'une des trois caractéristiques formelles de la phrase conditionnelles, soit l'ordre fixe protase - apodose, l'absence de conjonction de subordination ou la présence d'un morphème du conditionnel dans la protase.

3.1. Les constructions qui ne suivent pas l'ordre protase - apodose. L'ordre protase - apodose des phrases conditionnelles correspond en dzùùngoo à l'ordre chronologique dans lequel se posent les différentes propositions. L'ordre chronologique étant plutôt stricte dans l'organisation du discours, l'ordre protase - apodose ne souffre pas de variation. Les quelques phrases qui présentent une exception méritent examen.

Un premier exemple n'est pas vraiment une phrase conditionnelle puisque la subordonnée qui contient l'expression de la condition, c'est-à-dire la protase, n'est pas au conditionnel mais au subjonctif. La protase suit l'apodose.

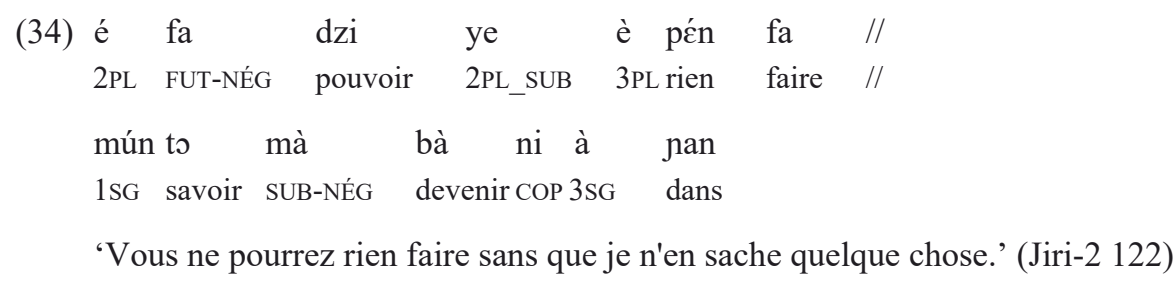

Dans cet exemple, on peut argumenter que la protase n'exprime pas vraiment une condition mais plutôt une cause. On reconnaît toutefois dans une telle phrase l'expression d'une virtualité d'actualisation.

Dans un autre exemple, le conteur utilise la postposition bóshjùn "sauf, sans" à la fin d'une protase isolée dont l'apodose se situe dans la phrase précédente.

(35) a. wó cíà rì̀ kosıbe // fó e vwèc̀y // 2SG briser_IAC FOC beaucoup // jusqu'à 2SG_SUB courir //

'On te frappe exagérément jusqu'à ce que tu fuies.' (Colonisation 255) 
b. mòò ye ná cínán a ma bóshòùn

personne COND venir aider 2SG à sauf

'Sauf si quelqu'un te vient en aide.' (Colonisation 256)

Dans cet exemple, la protase est marquée normalement pas le morphème du conditionnel ; la postposition bósh̀̀ùn "sauf" se positionne en final de la proposition comme si c'était un constituant nominal.

Dans un autre exemple, la protase, au lieu de la précéder, est incorporée dans l'apodose :

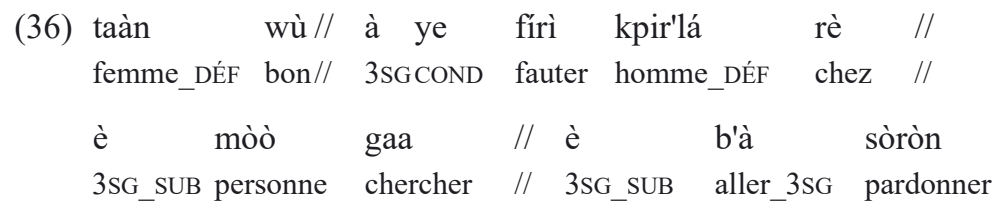

'La femme doit, si elle est en faute avec son mari, chercher quelqu'un pour aller lui demander pardon.' (Mariage 177)

Dans cet exemple, la protase aurait dû se placer devant le sujet de l'apodose ; l'apodose exprimant une modalité déontique, elle consiste en une structure syntaxique complexe avec une subordonnée (ici deux, à la deuxième ligne de l'exemple (36)) qui suit une principale. L'incorporation de la protase entre la principale de l'apodose et les deux propositions qui lui sont subordonnées répond à un effet de style permettant au narrateur de marquer une pause emphatique au milieu de l'énonciation d'un point important pour lui.

3.2. Les constructions qui utilisent une conjonction de subordination. Le bilinguisme des Dzùùns en jula est très élevé, et comme cela arrive dans beaucoup de langues de la région, les emprunts au jula sont très nombreux. Le système numéraire du dzùùngoo en particulier est en train de disparaître, remplacé par celui du jula ; chez certains, la structure des concessives du jula vient aussi se calquer sur celle du dzùùngoo (cf. point 2.5 ci-dessus) ; l'expression de la condition aussi prend chez certains locuteurs les formes du jula, sans le morphème du conditionnel et avec la conjonction $n i$ empruntée à cette langue. Comme en jula, le mode de toute la phrase est indicatif et le verbe de la protase est à l'aspect accompli.

$\begin{array}{llllllll}\text { (37) è } & \text { fá } & \text { dzi } & \text { dòn kòò re } & & \\ \text { 3PL } & \text { FUT-NÉG } & \text { pouvoir } & \text { entrer trou chez } & & \\ \text { ni } & \text { è na } & \text { vyèvyèć } & \text { vyè } & \text { bó } & \text { shía } & \text { re } & \text { waa } \\ \text { si } & \text { 3PL NÉG } & \text { toile_DÉF } & \text { déchirer } & \text { sortir } & \text { route_DÉF } & \text { chez } & \text { NÉG }\end{array}$

'... ils n'auraient pas pu entrer dans le trou s'ils n'avaient pas déchiré la toile d'araignée et libéré le passage.' (Maadu 83)

L'exemple (37) ci-dessus présente une structure inversée Q apodose - P protase ; la condition exprimée est irréelle irréalisée ; la phrase est négative, autant Q que P ; on retrouve dans l'apodose la particule fá de la négation du futur mais pas la copule ni de la structure typique (voir point 2.9). 
L'emploi de la conjonction $n i$ du jula s'accompagne d'une forme verbale de la protase à l'accompli, comme en jula. Une telle construction est rare dans les textes narratifs ; elle est plutôt typique des usages parlés des Dzùùns familiers avec l'emploi du jula dans les échanges commerciaux. Mais des conteurs aussi se laissent influencer par les usages du jula, en particulier si les histoires racontées ont été premièrement apprises dans cette langue, comme ce doit être le cas avec cet exemple tiré d'un enseignement de la tradition musulmane.

3.3. Les constructions qui n'utilisent pas de morphème du conditionnel. Nous avons pu constater déjà que des constructions subordonnées au subjonctif pouvaient avoir un sens d'irréel. La traduction en français de certaines phrases peut faire penser qu'il s'agit de phrases conditionnelles parce que la subordonnée est antéposée par rapport à la principale ; l'absence du morphème du conditionnel et la présence du subjonctif font plutôt penser qu'il s'agit d'antéposition de complétives par thématisation :

\begin{tabular}{|c|c|c|c|c|c|c|c|}
\hline (38) & $\begin{array}{l}\text { mòò } \\
\text { personne }\end{array}$ & $\begin{array}{l}\text { yè nà } \\
\text { suBvenir }\end{array}$ & $\begin{array}{l}\text { dòn gàné } \\
\text { entrer lieu }\end{array}$ & $\begin{array}{ll}\text { ròn } / / & \text { tò } \\
\text { à } & / / \\
\text { DÉM }\end{array}$ & $\begin{array}{l}\text { yéè } \\
\text { SuB }\end{array}$ & $\begin{array}{l}\text { bó } \\
\text { sortir }\end{array}$ & $\begin{array}{l}\text { kón // } \\
\text { encore // }\end{array}$ \\
\hline & mù & in fá & dzi & e tsiì & tò & ra wa & \\
\hline & ah! 1PL & FUT-NÉC & pouvoir & RÉF accepter & DÉM & à NÉG & \\
\hline
\end{tabular}

'Quelqu'un qui finit par entrer chez nous, s'il faut qu'il ressorte encore, nous ne pouvons pas accepter cela.' (Jiri-2 158)

Les deux subordonnées de la première ligne font penser à des conditionnelles; en fait ce sont des complétives, compléments du verbe accepter ; normalement, elles devraient se trouver en position post-verbe ; comme elles sont elles-mêmes liées entre elles, elles sont mieux placées antéposées.

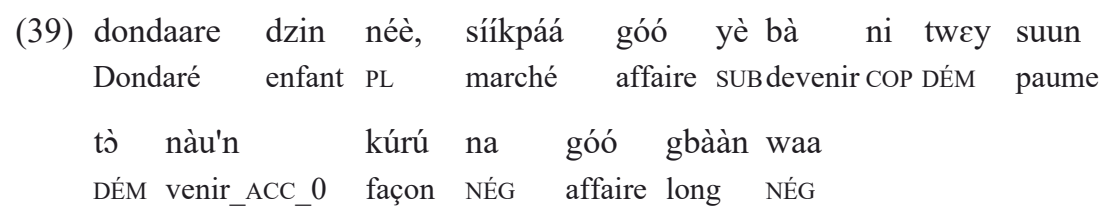

'Ceux de Dondaré, si le marché est entre leurs mains, cela ne leur est pas arrivé depuis longtemps.' (Jiri-2 147)

La phrase de ce dernier exemple figure à l'introduction d'un nouveau point dans une narration ; la construction fait penser à celle présentée au point 2.4 plus haut. La seule différence est l'utilisation du subjonctif au lieu du conditionnel ; on peut aussi poser l'hypothèse d'une complétive sujet de la principale de la deuxième ligne, reprise par un pronom démonstratif rappel t’̀.

\section{Conclusion}

Nous avons pu nous rendre compte en nous penchant sur les phrases conditionnelles du dzùùngoo que l'examen d'une telle problématique ouvrait sur plusieurs autres problématiques à savoir, la 
variété des usages d'un morphème verbal comme le conditionnel, la variété des constructions permettant l'encodage de la condition et la variété des constructions permettant l'encodage de l'irréel.

Ainsi, le morphème du conditionnel dans sa double forme affirmative et négative, en plus de caractériser l'expression explicite d'une condition intervient aussi dans l'expression de la cause (point 2.1) ou du temps (point 2.2) mais aussi dans l'expression de l'incertitude (point 2.3), dans l'organisation du discours comme procédé de thématisation, dans l'introduction d'un nouvel argument (point 2.4) ou dans la construction d'un tête-à-queue (point 2.5), ainsi que dans l'expression de la concession (points 2.6 et 2.8 ).

Le morphème du subjonctif dans sa double forme affirmative et négative n'est pas non plus étranger à l'expression de la condition puisqu'il peut intervenir dans les constructions conditionnelles et concessives calquées sur les structures du jula (points 2.8 et 3.2) ; il intervient encore dans des complétives antéposées qui peuvent fonctionner comme des conditionnelles (point 3.3).

De toutes les nuances de conditions, il n'est que celle de l'irréel, irréalisable ou irréalisé, qui soit nettement encodée. Que ce soit dans l'apodose des structures complexes ou dans les phrases simples, l'irréel affirmatif est encodé par combinaison du morphème du passé ná associé au morphème du futur $n a ̀$; le négatif par les morphèmes futur négatif fá et copule $n i$. Au niveau de la protase, l'expression de l'irréel dans l'apodose semble être cooccurrente de formes verbales en ye bá ná 'COND devenir PAS'. Des variantes stylistiques semblent aussi possibles dans l'encodage des formes verbales autant de la protase (exemple (33)) que de l'apodose (exemples (31 \& (32)).

\section{Tableau des abréviations}

$\begin{array}{llll}1 & \text { Première personne } & \text { lit. } & \text { Littéralement } \\ 2 & \text { Deuxième personne } & \text { LOG } & \text { Pronom logophorique } \\ 3 & \text { Troisième personne } & \text { NEG } & \text { Négatif } \\ \text { ACC } & \text { Aspect accompli } & \text { PART } & \text { Participe passé } \\ \text { COND } & \text { Conditionnel } & \text { PAS } & \text { Passé } \\ \text { COND-NEG } & \text { Conditionnel négatif } & \text { PL } & \text { Pluriel } \\ \text { COP } & \text { Copule } & \text { POSS } & \text { Possessif } \\ \text { DEF } & \text { Défini } & \text { REF } & \text { Pronom réfléchi } \\ \text { DEM } & \text { Démonstratif } & \text { REL } & \text { Pronom relatif } \\ \text { FOC } & \text { Focalisateur } & \text { RET } & \text { Aspect rétrospectif } \\ \text { FUT } & \text { Futur } & \text { SG } & \text { Singulier } \\ \text { FUT-NEG } & \text { Futur négatif } & \text { SUB } & \text { Subjonctif } \\ \text { IAC } & \text { Aspect inaccompli } & \text { SUB-NEG } & \text { Subjonctif négatif } \\ \text { ICH } & \text { Aspect inachevé } & \text { / } & \text { Limite grammaticale de propositions }\end{array}$




\section{Références}

Feuillet, Jack. 2006. Introduction à la typologie linguistique. Paris: Honoré Champion.

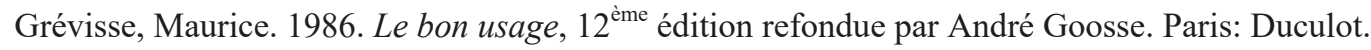
Haiman, John. 1978. Conditionals are topics. Language 54: 564-589.

Kastenholz, Raimund. 2003. "Samogo" language islands, and Mande-Senufo (Gur) interference phenomena, in Lexical and structural diffusion, éd. par R. Nicolai et P. Zima. (CORPUS, Les Cahiers, 1). pages 91-110, Nice: Publications de la Faculté des Lettres de Nice.

Solomiac, Paul. 2014. Phonologie et morphosyntaxe du dzùùngoo de Samogohiri. Köln: Rüdiger Köppe Verlag.

\begin{tabular}{|l|}
\hline Paul Solomiac \\
paul_solomiac@sil.org \\
\hline
\end{tabular}

Submitted: 31 August 2015

Accepted: 11 March 2016

Revisions: 27 April 2016 